\title{
The Neuroid revisited: A heuristic approach to model neural spike trains
}

\author{
Erick Javier Argüello Prada ${ }^{1 *}$, Ignacio Antonio Buscema Arteaga ${ }^{2}$, Antonio José D’Alessandro Martínez ${ }^{3}$ \\ ${ }^{1}$ Department of Information Technology and Communications, Faculty of Engineering, Santiago de Cali University, Valle del \\ Cauca, Colombia. \\ ${ }^{2}$ Departament of Technological, Biological and Biochemical Processes, Simón Bolivar University, Caracas, Venezuela. \\ ${ }^{3}$ Myocardial Contractility Laboratory, Institut of Experimental Medicine, Faculty of Medicine, Central University of Venezuela, \\ Caracas, Venezuela.
}

\begin{abstract}
Introduction: Since it was introduced in 2012, the Neuroid has been used to aid in understanding how functionally different neural populations contribute to sensory information processing. However, insights about whether this neuron-model could perform better than others or about when its utilization should be considered have not been provided yet. Methods: In an attempt to address this issue, a comparison between the Neuroid and the leaky-integrate-and-fire (LIF) model in terms of accuracy and computational cost was performed. Both models were tested for different stimulation amplitudes and stimulation periods, with time step sizes ranging from $10^{-4}$ to $1 \mathrm{~ms}$. Results: It was found that, although the Neuroid was able to produce more accurate results than its original version, its accuracy was lower than the achieved with the LIF model solved by the forward Euler method. On the other hand, the Neuroid performed its calculations in an amount of time significantly lower (Mulfactorial ANOVA test, $\mathrm{p}<0.05$ ) than that required by the LIF model when it was solved by using the forward Euler method. Moreover, it was possible to use Neuroid-based networks to replicate biologically relevant firing patterns produced by low-scale networks composed of more detailed neuron-models. Conclusion: Results suggest that the Neuroid could be an interesting choice when computational resources are limited, although its use might be restricted to a narrow band of applications.
\end{abstract}

Keywords Neuroid, Spiking neuron-model, Frequency-intensity curve, Accuracy, Computational cost, Heuristic.

\section{Introduction}

In spite of their simplicity, phenomenological models of spiking neurons have proved particularly useful in elucidating the dynamics of single neurons, as well as their role within large-scale neural networks. For instance, it was found that integrate-and-fire models with adaptation could predict the response of cortical neurons to somatically injected currents with high accuracy in spike timing (Brette and Gerstner, 2005; Jolivet et al., 2004; Kobayashi et al., 2009). An innovative alternative known as Neuroid (Prada et al., 2012) was introduced at the $34^{\text {th }}$ International Annual Conference of the IEEE Engineering in Medicine and Biology Society

(c) This is an Open Access article distributed under the terms of the Creative Commons Attribution License, which permits unrestricted use, distribution, and reproduction in any medium, provided the original work is properly cited.

How to cite this article: Prada EJA, Arteaga IAB, MartínezAJD. The Neuroid revisited: a heuristic approach to model neural spike trains. Res Biomed Eng. 2017; 33(4):331-343. DOI: 10.1590/2446-4740.02617.

*Corresponding author: Departamento de Tecnologías de la Información y las Comunicaciones, Universidad Santiago de Cali, Calle 5 \# 62-00 Barrio Pampalinda, Santiago de Cali, Valle del Cauca, Colombia. E-mail: erick.arguello00@usc.edu.co

Received: 27 May 2017 / Accepted: 23 November 2017
(EMBC'12). Since then, this neuron-model has been utilized to aid in understanding how functionally different neural populations (e.g., low-threshold vs. high-threshold afferent fibers; normally silent vs. tonically active neurons) interact each other and how those differences affect the way that visual (Prada et al., 2013) and tactile (Prada and Bustillos, 2013; Prada et al., 2015) information is processed.

The Neuroid is, indeed, one of the simplest neuron-models that have ever been conceived. It was built under the assumption that some neurons, under certain conditions, fire at regular rates when stimulation amplitude is held constant. The similarities between the action potential generation process and pulse frequency modulation (PFM) that have been pointed out by several authors (Bayly, 1968; Horch and Dhillon, 2004; Rieke et al., 1997) were also considered in building this neuron-model. It relies on a pair of heuristics that make possible to generate a spike train whose frequency is modulated by the amplitude of the stimulus (even though is widely known that the neuron firing rate may be affected by other factors, like the sub-threshold dynamics) and to demodulate the spike train in order to recover the modulating signal. But the real novelty of the Neuroid lies on what kind of experimental data is used to model different neural 
populations. Instead of ionic conductances, equilibrium potentials and/or electrical membrane properties, the firing rate as a function of the stimulus intensity is the only requirement to build a Neuroid-based replica of the neuron under study. Essentially, the goal is to adjust the Neuroid's parameters such that its frequency-intensity (F-I) curve resembles as much as possible the one that was built from experimental data. This novel approach, although simple in concept and application, may be little confusing for those who have already dealt with the

issues involved in using conductance-based neuron-models. Likewise, insights about whether this neuron-model could perform better than others or about when its utilization should be considered have not been provided so far.

Coordinated rhythmicity of neural populations may arise from the interaction of three aspects: the intrinsic properties of the cells composing the network, the synaptic properties of connections between neurons, and the topology of the network (Ermentrout and Terman, 2010). Specifically, the rhythm with which vertebrates and invertebrates walk, fly or swim, as well as communication based in stereotypically repeated visual or sound signals (e.g., the cricket song), is mostly driven by oscillatory patterns produced by neural networks of the central nervous system (Delcomyn, 1980). In order to study the mechanisms underlying this oscillatory activity, several authors (Friesen and Stent, 1977; Kling and Székely, 1968; Koch and Brunner, 1988) have used artificial neurons, also known as "neuromimes", built from elementary electronic components and able to provide a biologically plausible description of the neuron's membrane. Nevertheless, it is unclear whether that activity is due to one of the above aspects, or to their combined action. Moreover, it is unknown whether oscillatory patterns produced by low-scale networks composed of highly detailed neuron-models can be replicated by Neuroid-based replicas of those networks.

In this sense, the present study aims: 1) to study how different sizes of time step affect the accuracy of the Neuroid, 2) to compare its performance to the exhibited by the leaky-integrate-and-fire (LIF) model in terms of accuracy and computational cost, and 3) to assess the feasibility in using Neuroid-based networks to replicate biologically relevant firing patterns produced by low-scale networks composed of much more detailed neuron-models.

\section{Methods}

\section{Theoretical background}

It is widely accepted that stimulus information (e.g., the intensity) is encoded not in the magnitude of a single spike but in the number of spikes fired per unit time (firing rate). With that in mind, we think it would be appropriate to provide a mathematical representation of neural spike trains rather than investing time and effort in describing the action potential as an isolated event, as brilliantly done by Hodgkin and Huxley (Hodgkin and Huxley, 1952). Let us consider the triggering event $s(t)$ given by:

$$
s(t)=\sum_{j=1}^{M} w_{j} x_{j}(t),
$$

where $x_{1}, x_{2}, \ldots, x_{M}$ are $M$ normalized input signals and $w_{1}, w_{2}, \ldots, w_{M}$ are their respective synaptic weights. Then, based on the fact that, under specific conditions, some neurons can fire at a regular rate when the stimulus amplitude remains constant, the signal travelling along the neuron's axon $y(t)$ can be modeled as:

$$
y(t)=\left\{\begin{array}{cc}
\sum_{n=0}^{\infty} \delta\left(t-\frac{n \beta T}{s(t)-\vartheta}\right) & \text { if } s(t)>\vartheta \\
0 & \text { otherwise }
\end{array}\right.
$$

where $\delta(\cdot)$ represents a single spike, $T$ denotes a constant with time units (necessary for dimensional consistency), $\vartheta$ is the relative threshold intensity and $\beta$ is a proportionality constant that controls the slope of the Neuroid's (F-I) curve. The Neuroid is also able to yield an amplitude-discrete version of $s(t)$, named $n t$ out $(t)$, by adjusting two additional parameters: $K_{r}$, a dimensionless constant which controls the output's amplitude, and maxcount, a temporal parameter which allows to extend the output signal by several milliseconds after the last spike (Prada et al., 2012).

Even though it is not evident from the present study, each parameter of the Neuroid is inspired by a biological property of real neurons. For instance, the term $\vartheta$ represents the minimum stimulus intensity at which the neuron fires, at least, one action potential, and it has been defined in this way in order to be consistent with different stimulus types (thermal, mechanical, etc). The parameter $\beta$ is associated to the potassium current flowing through the membrane, so the greater its value, the lower the slope of the F-I curve (Alberts et al., 2002; Koch and Brunner, 1988). The term maxcount denotes the time during which the neurotransmitter concentration is such that it has a synaptic effect in the cleft (Scimemi and Beato, 2009). Finally, although the parameter $K$ does not have an obvious biological counterpart, its value depends on the firing frequency at the maximum amplitude of stimulation, which is calculated from the experimental F-I curve.

To avoid the division by zero in Equation 1, it was arranged that spike trains were generated only if the amplitude of the triggering input signal $s(t)$ exceeds the threshold intensity $\vartheta$. However, this is not consistent with experimental observations, which underline that spikes are produced not only when neuron's membrane 
potential exceeds threshold, but also when the former reaches the latter (Matzner and Devor, 1992). To address this issue, the heuristic able to produce the signal $y(t)$ (the Pulse Frequency Modulator in (Prada et al., 2012) was modified such that the case in which the membrane potential reaches threshold was considered. Figure 1 shows a flowchart describing the new heuristic.

The accuracy of a neuron-model describes how reliable is the model in representing the behavior of biological neurons, in terms of the temporal resolution with which neural spikes can be produced (from miliseconds to fractions of miliseconds). Since simulating spiking neuron-models involves repeatedly solving the associated system of differential equations using analytical or numerical integration methods, the accuracy provided by such models may depends on, amongst other factors, the numerical method used to solve the system and the size of the time step $\Delta t$, an aspect that has not been explicitly mentioned and whose influence has not been studied in previous implementations of our neuron-model.

\section{Modeling approach}

For each $I \in\left[I_{\min }, I_{\max }\right]$, where $I_{\min }$ and $I_{\max }$ denote, respectively, the minimum and the maximum amplitudes of stimulation, there is an $x \in[0,1]$ given by:

$x(t)=\frac{I(t)-I_{\min }}{I_{\max }-I_{\min }}$, which represents one of the multiple inputs that may contribute to create the triggering event $s(t)$, as expressed in Equation 1. As can be seen from Figure 1, if $s(t)>\vartheta$, a spike is fired whenever the variable count 1 reaches the smallest integer greater than or equal to the quantity $\frac{\beta}{(s(t)+\vartheta) \Delta t}$. Thus, the interspike interval (ISI) of the spike train yielded by the Neuroid for a given $s(t)$, can be calculated by using Equation 4:

$I S I=\lceil\beta / s(t) \Delta t+1\rceil \Delta t$,

where the operator $\lceil\cdot\rceil_{\text {denotes the }}$ ceiling function, which is defined as follows:

$\lceil r\rceil=\min \{p \in \mathrm{Z} \mid p-1<r \leq p\}$,

where $r$ is a real number.

From Equation 5, it is clear that for all real number $r$ we have:

$\lceil r\rceil \geq r$.

Therefore, from Equations 4 and 6, the value of the parameter $\beta$ can be expressed as:

$\beta \leq\left(\frac{I S I}{\Delta t}-1\right) s(t) \Delta t$.

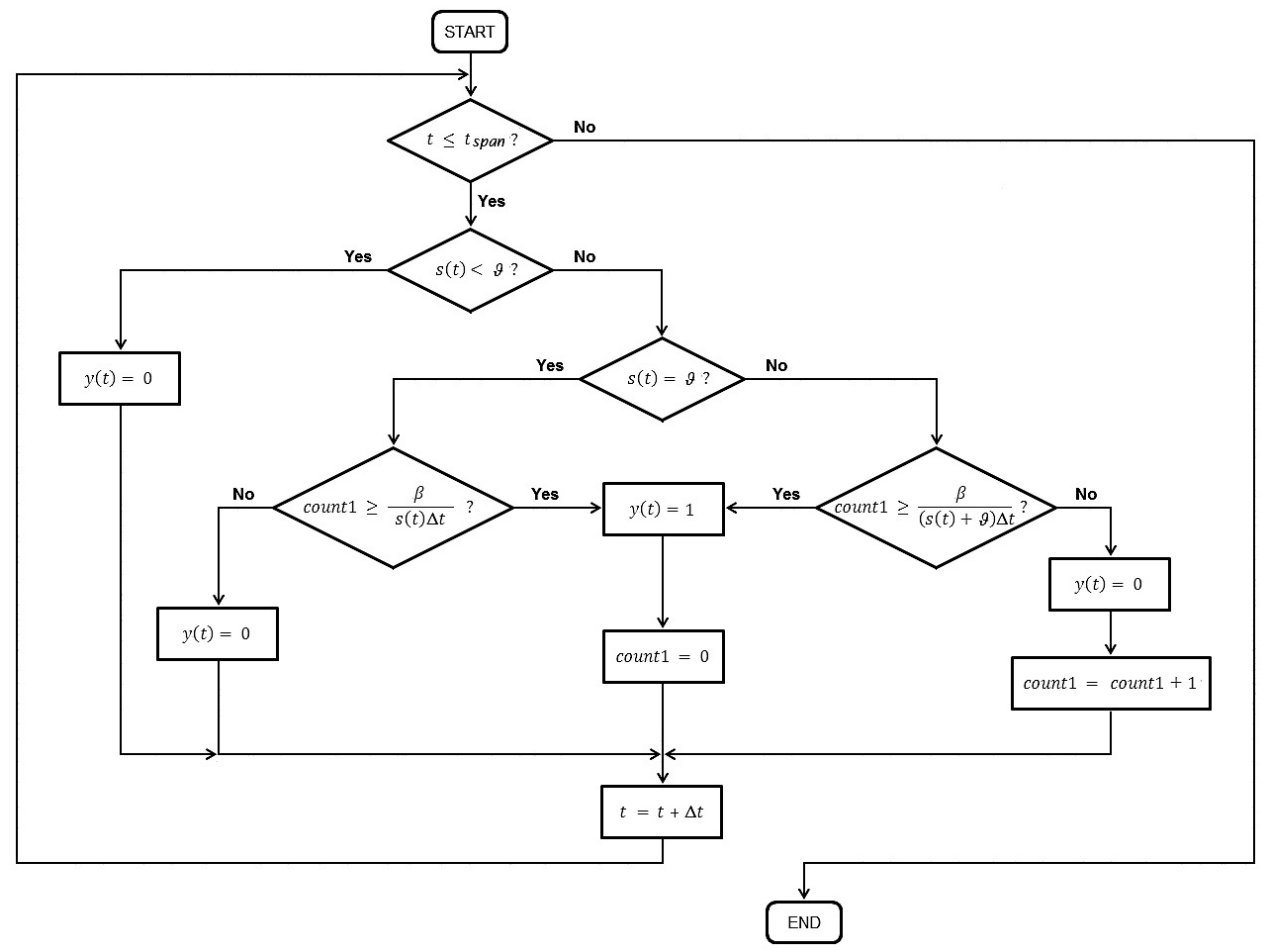

Figure 1. A novel heuristic, which, unlike the one originally proposed by Prada et al. (2012), considers the case in which membrane potential reaches threshold. 
Typically, the firing rate $(f)$ of a single neuron is calculated by using Equation 8:

$$
f=\frac{N_{\text {spikes }}}{t_{\text {last }}-t_{\text {first }}},
$$

where $N_{\text {spikes }}$ is the total number of spikes fired within the observation period while $t_{\text {frrst }}$ and $t_{\text {last }}$ are, respectively, the instants at which the first and the last spike occur.

Interestingly, Equation 8 can be rewritten as:

$$
f=\frac{N_{\text {spikes }}}{\left(N_{\text {spikes }}-1\right) I S I_{\text {avg }}},
$$

where $I S I_{\text {avg }}$ represents the average interspike interval, which can be approximated to ISI when the neuron fires at a regular rate.

Finally, by identifying the firing rate of the neuron under study at the maximum amplitude of stimulation $\left(f_{I_{\max }}\right)$, the interspike interval at that amplitude $\left(I S I_{I_{-\max }}\right)$ is given by:

$$
I S I_{I_{-} \max }=\frac{N_{\text {spikes }}}{\left(N_{\text {spikes }}-1\right) f_{I_{-} \max }},
$$

which in turn allows us to rewrite Equation 7 to calculate the value of $\beta$ required to model that neuron (note that $s(t)=1$ at the maximum amplitude of stimulation):

$$
\beta \leq\left(\frac{I S I_{I_{-} \max }}{\Delta t}-1\right) \Delta t .
$$

\section{Evaluating the Neuroid's performance: firing pattern and F-I curve}

To evaluate the performance of a neuron-model, a benchmark solution (i.e., another model capable of providing a fairly good representation of neuronal behavior) is required. In our case, the benchmark solution was built by implementing the LIF model (Jolivet et al., 2004), which is commonly described by Equation 12:

$$
\tau_{m} \frac{d V(t)}{d t}=E_{\text {rest }}-V(t)+R_{m} I(t), \text { if } V(t) \geq V_{\text {th }} \text { then } V(t) \rightarrow V_{r}
$$

where $V(t)$ is the membrane potential, $I(t)$ is the input current, $E_{\text {rest }}$ is the resting membrane potential, $V_{t h}$ is the firing threshold, $V$ is the after-spike membrane potential, $R_{m}$ is the membrane resistance and $\tau_{m}$ is the membrane time constant. The LIF model was chosen as the benchmark solution because it has only a small number of parameters and it is one of the most computationally efficient spiking neuron-models, as reported in previous work (Long and Fang, 2010; Skocik and Long, 2014). In order to compute the parameters required to build a Neuroid-based replica of this "neuron", Equation 12 was numerically solved by adopting the fourth-order Runge-Kutta method (RK4) with a time step $\Delta t=10^{-4} \mathrm{~ms}$. The benchmark solution was implemented under MatLab 2013a using
$E_{\text {rest }}=0 \mathrm{mV}, V_{\text {th }}=30 \mathrm{mV}, V_{r}=-50 \mathrm{mV}, V_{\text {spike }}=70 \mathrm{mV}$, $R_{m}=8.22 \mathrm{M} \Omega, \tau_{m}=23.5 \mathrm{~ms}$, and its F-I curve was built by increasing the amplitude of a rectangular current pulse $I(t)$ applied during $1000 \mathrm{~ms}$ (the total time of run of each trial, denoted as $\left.t_{\text {span }}\right)$, from 0 to $20 \mathrm{nA}$ (increments of $0.1 \mathrm{nA}$ ). The frequency was calculated by using Equation 8 .

Once the F-I curve of the benchmark solution was built, the threshold intensity $\left(I_{t h}=3.7 \mathrm{nA}\right)$ and the firing rate at the maximum amplitude of stimulation $\left(f_{I \max }=92.11 \mathrm{~Hz}\right)$ were identified. Then, to build a Neuroid-based replica of that "neuron", we found $\beta$ from Equation 11 whereas $\vartheta$ was calculated by using Equation 13:

$\vartheta=\frac{I_{t h}-I_{\min }}{I_{\max }-I_{\min }}$,

where $I_{t h}$ is the threshold intensity.

Both the Neuroid $\left(\Delta t=10^{-4} \mathrm{~ms}\right)$ and the benchmark solution were run in MatLab 2013a for $I=3.7 \mathrm{nA}$ (the threshold intensity) and $I=6 \mathrm{nA}$ to compare their firing patterns at this two stimulation amplitudes. The same method used to calculate the firing rate of the LIF model, was used to calculate the firing rate achieved by the Neuroid. With time steps ranging from $10^{-4}$ to $1 \mathrm{~ms}$, the F-I curve of the Neuroid was compared to the one previously built for the benchmark solution.

\section{Accuracy and computational cost}

Several methods have been used recently to evaluate the accuracy of a neuron-model. Wang and coworkers (Wang et al., 2014) totalized the number of false negatives (missed spikes) and false positives (additional or accidental spikes) with respect to the spike train generated by the HH neuron-model solved by the RK4 method, and then they divided each total by the number of matched spikes pairs to obtain two different indicators of accuracy. Skocik and Long (2014) estimated the accuracy of the LIF model, the HH model and the Izhikevich's model (Izhikevich, 2003) by quantifying the absolute difference between the frequency of the model being tested $\left(f_{\text {model }}\right)$ and the frequency of its respective benchmark solution $\left(f_{B M}\right)$, which was taken to be the solution provided by the RK4 method with a time step of $10^{-4} \mathrm{~ms}$, and then dividing the difference by the latter, as expressed in Equation 14:

$$
\text { Frequency Error }(\%)=\frac{\left|f_{\text {model }}-f_{B M}\right|}{f_{B M}} \times 100 \text {. }
$$

Such estimation was performed for three different frequency values $(70,90$ and $120 \mathrm{~Hz}$ ), which were considered as falling into the low-, medium-, and high-frequency range. Since these ranges may vary between different neuronal populations, we estimated the accuracy of the Neuroid by calculating the frequency 
error defined by Skocik and Long (2014), except that this time the estimation was performed for three different stimulation amplitudes, taking into account the relative location of these amplitudes with respect to the dynamic range of the benchmark solution (i.e., the range of amplitudes for which it is able to fire, from $I_{t h}$ to $\left.I_{\max }\right)$. Thus, three values were chosen: one slightly greater than the threshold intensity $(I=5 \mathrm{nA})$, one at the middle of the dynamic range $(I=12 \mathrm{nA})$, and one close to the maximum stimulation amplitude $(I=19 \mathrm{nA})$. For each, with a total time of run $t_{\text {span }}=1000 \mathrm{~ms}$ and with time steps ranging from $10^{-4}$ to $1 \mathrm{~ms}$, we quantified the frequency error produced by both the Neuroid and the LIF model solved by the Forward Euler (FE) method.

The interest in calculating the computational cost of a neuron-model arises from the assumption that the less computational power is required to simulate a single neuron, the easier it is to implement it into a large-scale neural network. According to several authors (Izhikevich, 2004; Long and Fang, 2010; Skocik and Long, 2014), a good indicator of how fast a neuron-model will perform its calculations is the number of floating point operations (FLOPs) required by the model. For a given time of run $\left(t_{\text {span }}\right)$, the total of FLOPs required by a neuron-model can be estimated as follows:

Total FLOPs $=(\#$ FLOPs $)(\#$ iterations $)$,

where AFLOPS denotes the number of FLOPs per iteration and \#iterations represents the total number of iterations for the given time of run:

$\#$ iterations $=\frac{t_{\text {span }}}{\Delta t}$.

The number of FLOPs per iteration depends mostly on the method used to determine if a spike can or cannot be fired. For instance, when the FE method is used to solve the LIF model (see Equation 12), the membrane potential is updated as follows:

$V(t+\Delta t)=V(t)+\left(E_{\text {rest }}-V(t)+R_{m} I(t)\right)\left(\frac{\Delta t}{\tau_{m}}\right)$,

which takes only 5 FLOPs by assuming that addition, multiplication, subtraction and division costs only one FLOP each, as done in previous studies (Izhikevich, 2004; Long and Fang, 2010; Skocik and Long, 2014). However, from Figure 1, it can be seen that, depending on whether the triggering input signal $s(t)$ is less than, equal to or greater than the relative threshold intensity $\vartheta$, the number of FLOPs per iteration may vary, as expressed in Equation 18:

$\# F L O P S=\left\{\begin{array}{ll}0 & \text { if } s(t)<\vartheta \\ 3 & \text { if } s(t)=\vartheta, \\ x & \text { if } s(t)>\vartheta\end{array} \quad\right.$ wherex $= \begin{cases}2 & \text { if a spikeis fired } \\ 3 & \text { if no spikeis fired }\end{cases}$
This suggests that the total of FLOPs required by the Neuroid for a given time of run may decrease 1) when no stimulus is present, and 2) when firing rate increases. To test this hypothesis, we run both the Neuroid and the LIF model solved by the FE method under Dev-C++4 4.9.9.2, an integrated development environment for the $\mathrm{C} / \mathrm{C}++$ programming language, with time steps ranging from $10^{-4}$ to $1 \mathrm{~ms}$. We used the $\mathrm{FE}$ method instead of the RK4 method because, even though the former is not the most accurate, it is one of the simplest numerical methods (Skocik and Long, 2014), which allowed us to perform a much more fair comparison. Wang et al. (2014) introduced a generalized exponential moving average method to solve a modified version of the LIF model. Such method required a computation time lower than the required by the original LIF model and the HH model when they were solved by adopting the RK4 method. On the other hand, the utilization of the RK4 method for benchmarking clearly favored the method proposed by the group of Wang because the former is computationally expensive. Instead, we used a numerical method more affordable from a computational point of view in order to make a more fair comparison. $\mathrm{The} \mathrm{C}++$ programming language was used in calculating the computation time required by the Neuroid and the LIF model because it is possible to speed-up simulations up to several hundreds of times compared to MatLab (Andrews, 2012).

With time steps ranging from $10^{-4}$ to $1 \mathrm{~ms}$, the LIF model and the Neuroid were run for three different simulation amplitudes $(I=5,12$ and $19 \mathrm{nA})$ and for three different stimulation periods ( $T=200,500$ and $800 \mathrm{~ms}$ ). The computation time required by each model was measured by using the QueryPerformanceCounter() function, and measurements were averaged over 10 repetitions. A factorial design was adopted by considering the neuron-model (LIF and Neuroid), the time step size, the stimulus amplitude and the stimulation duration as factors, each with 2, 5, 3 and 3 possible values or levels, respectively. Even though the analysis of the effects of interactions between factors on the computation time achieved by each model is beyond the scope of the present study, a multi-factor analysis of variance (ANOVA) was used to identify significant effects of each factor on the computation time (Montgomery, 2017). Values of $p<0.05$ were considered statistically significant. Microsoft Excel 2010 (Microsoft Corporation, USA) and STATGRAPHICS Centurion XVI.I (version 16.1.18, Statistical Graphics Corporation) and Minitab 18 (ver 18.1, Minitab, Inc.) were used to perform the analysis.

\section{Low-scale neural networks}

To assess whether Neuroid-based networks are able to preserve oscillatory patterns produced by networks composed of those artificial units, we replicated the 
topology of the three-neuron oscillator and the "cricket song generator", previously built by Koch and Brunner (1988) to test the neuron-model they developed. Neuroid-based replicas of the neurons modeled by Koch and Brunner were implemented under MatLab 2013a using $\vartheta=0.2, \beta=2.5, K_{r}=3$, maxcount $=24$ and $\Delta t=0.1 \mathrm{~ms}$ for the three-neuron oscillator, and $\vartheta=0.2$, $\beta=2.5, K_{r}=3$, maxcount $=24$ and $\Delta t=0.1 \mathrm{~ms}$ for the "cricket song generator". As done in previous studies (Prada and Bustillos, 2013; Prada et al., 2012; 2013; 2015), synaptic coupling factors were taken to be equal to 1 or -1 in order to simulate, respectively, postsynaptic facilitation or inhibition. However, in the "cricket song generator", neuron 4 strongly activates neuron 5, which in turn strongly inhibits neuron 1 (see section 3.2.4 in (Koch and Brunner, 1988)), so we empirically increased the coupling factors between these two pairs of neurons until the firing patterns of our Neuroid-based replica were as similar as possible to those obtained by Koch and Brunner. An Acer TravelMate B113, with an Intel Core i3-3217U and 4 GB of memory, was used to perform all simulations.

\section{Results}

\section{The Neuroid can now fire at the threshold intensity}

As shown in Figure 2, the Neuroid fired one single spike when the stimulation amplitude reached the threshold intensity $(I=3.7 \mathrm{nA})$, while the benchmark solution fired several spikes for the same stimulus. Both neuron-models showed a tonic response for $I=6 \mathrm{nA}$ and the total number of spikes produced by them were very similar.

\section{The accuracy of the Neuroid improved when a time step of $10^{-2}$ ms or lower was used}

A comparison of the F-I curves for the Neuroid and the benchmark solution is shown in Figure 3. With a time step of $1 \mathrm{~ms}$, discrepancies were observed between the responses produced by the Neuroid and the benchmark solution over the whole range of stimulation amplitudes. On the other hand, with a time step of $10^{-2} \mathrm{~ms}$, the response of the Neuroid was in good agreement with that provided by the benchmark solution, except for a short range of amplitudes slightly greater than the threshold intensity (from 3.7 to $7 \mathrm{nA}$ ). It is worth noting that, as with many neuron-models, the firing frequency calculated by the Neuroid "jumped" to a non-zero value instead of increasing continuously at the threshold intensity. In fact, at this value, the Neuroid yielded $f=0 \mathrm{~Hz}$ and only for stimulation amplitudes greater than threshold our neuron-model yielded non-zero frequency values. This is because, to calculate the firing frequency, at least two spikes were required (see Methods). Since at the threshold intensity, only one spike was fired, the condition was not fulfilled and the frequency was taken to be zero.

As it can be seen from Table 1, the Neuroid produced a firing frequency that approximates the one produced by the benchmark solution with an error less than $1 \%$, using a time step less than or equal to $10^{-2} \mathrm{~ms}$, except for $I=5 \mathrm{nA}$. Better results were obtained for the whole range of stimulation amplitudes by using the LIF model solved by adopting the FE method with a time step less than or equal to $10^{-1} \mathrm{~ms}$.

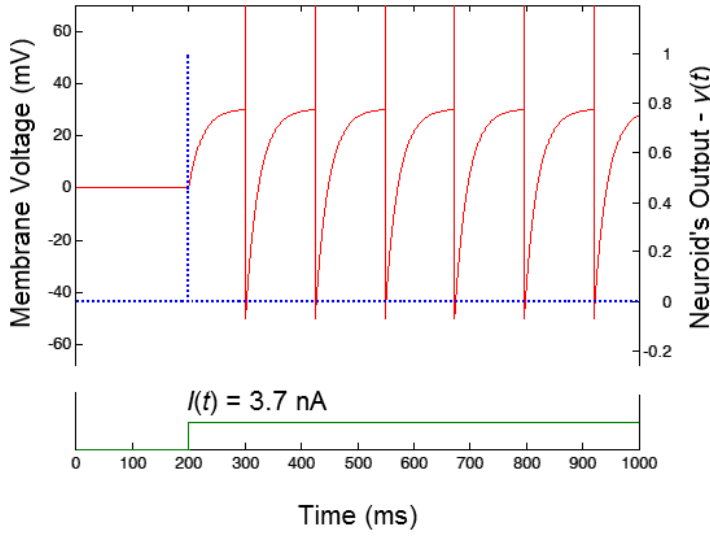

(a)

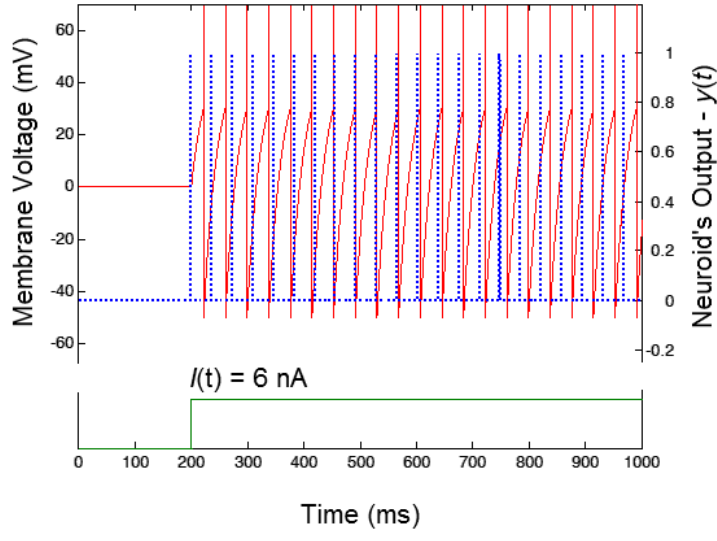

(b)

Figure 2. The firing pattern of the Neuroid $\left(\Delta t=10^{-4} \mathrm{~ms}\right)$ and the benchmark solution (the LIF model solved by the fourth order Runge-Kutta method with $\Delta t=10^{-4} \mathrm{~ms}$ ) for two different stimulation amplitudes: $3.7 \mathrm{nA}$ (threshold intensity) and $6 \mathrm{nA}$. (a) When the stimulation amplitude reached threshold, the Neuroid (blue dotted line) fired only one spike, while the benchmark solution (red solid line) fired several spikes. (b) For $I=6 \mathrm{nA}$ both models showed a tonic response, although the Neuroid added one more spike to the total produced by the benchmark solution. 


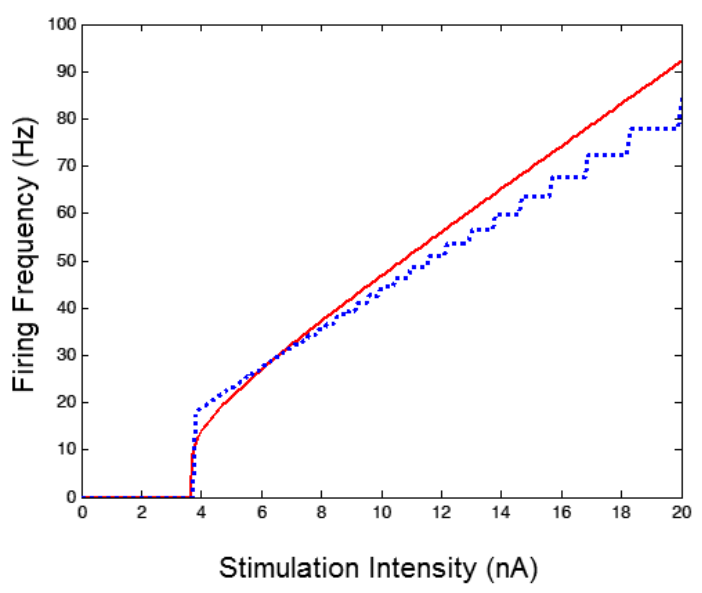

(a)

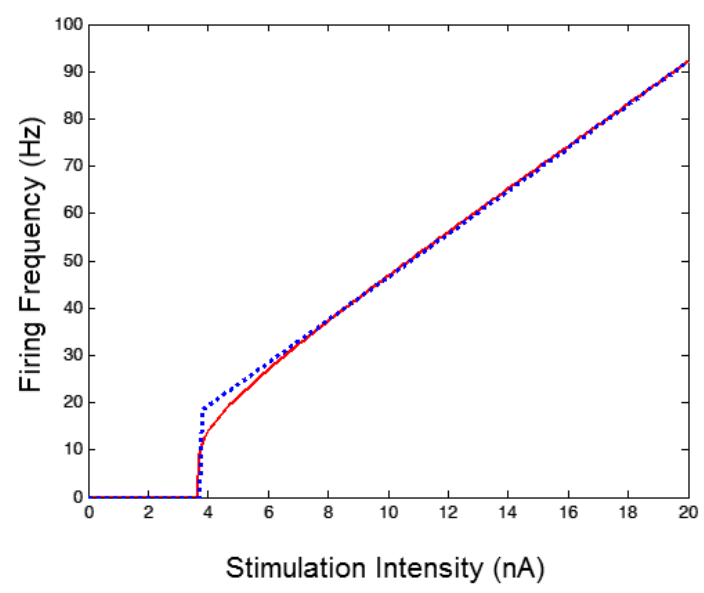

(b)

Figure 3. A comparison between the F-I curve of the Neuroid (blue dotted line) for two different time steps and the F-I curve of the benchmark solution (red solid line). (a) When a time step of $1 \mathrm{~ms}$ was used, the Neuroid showed a poor agreement with the benchmark solution. (b) When a time step of $10^{-2} \mathrm{~ms}$ (or lower) was used, the Neuroid showed a good agreement with the benchmark solution, except for a short range of amplitudes slightly greater than the threshold intensity.

Table 1. Frequency errors for the LIF model (solved by the Forward Euler method) and the Neuroid at three different stimulation amplitudes $(I=5,12$ and $19 \mathrm{nA})$ with time steps ranging from $10^{-4}$ to $1 \mathrm{~ms}$. Values are expressed in percentages.

\begin{tabular}{|c|c|c|c|c|c|c|}
\hline \multirow{3}{*}{$\begin{array}{c}\text { Time step } \Delta t \\
(\mathrm{~ms})\end{array}$} & \multicolumn{6}{|c|}{ Stimulation amplitude (nA) } \\
\hline & \multicolumn{2}{|c|}{5} & \multicolumn{2}{|c|}{12} & \multicolumn{2}{|c|}{19} \\
\hline & LIF & Neuroid & LIF & Neuroid & LIF & Neuroid \\
\hline $10^{0}$ & 0.94 & 9.16 & 0.84 & 9.09 & 3.80 & 11.10 \\
\hline $10^{-1}$ & 0.14 & 11.65 & 0.25 & 1.34 & 0.51 & 1.36 \\
\hline $10^{-2}$ & 0.00 & 11.89 & 0.02 & 0.78 & 0.01 & 0.26 \\
\hline $10^{-3}$ & 0.00 & 11.94 & 0.00 & 0.73 & 0.01 & 0.11 \\
\hline $10^{-4}$ & 0.00 & 11.94 & 0.02 & 0.73 & 0.00 & 0.10 \\
\hline
\end{tabular}

\section{The neuroid is significantly faster than the LIF model solved by the FE method}

From Figure 4 and Table 2, it can be seen that the mean computation time required by the Neuroid was significantly lower (Multifactorial ANOVA, $\mathrm{p}<0.05$ ) than the required by the LIF model when it was solved by using one of the simplest methods for approximating solutions to differential equations (i.e., the FE method). On the other hand, no significant differences were found in the computation time achieved by each model when different stimulation amplitudes and different stimulation periods were used (see Table 3 ).

Figure 5 shows, in the form of a Pareto chart, the absolute values of the standardized effects of each factor and all their possible combinations on the computation time required to calculate several hundreds of milliseconds of neural response. As can be seen, only the model (A) has a significantly and markedly effect on the computation time.
Table 2. Summary of multiple range tests for computation time (the response variable) by model. A multiple comparison procedure (the Fisher's least significant difference (LSD) procedure) was applied to determine which means are significantly different from which others. The bottom half of the table shows the estimated difference between means. An asterisk has been placed next to the pair showing a statistically significant difference at the $95 \%$ confidence level (LIF: leaky-integrate-and-fire; NEU: Neuroid).

\begin{tabular}{ccccc}
\hline Models & Count & LS Mean & $\begin{array}{c}\text { LS } \\
\text { Sigma }\end{array}$ & $\begin{array}{c}\text { Homogeneous } \\
\text { groups }\end{array}$ \\
\hline NEU & 450 & 31.4092 & 1.50113 & $\mathrm{X}$ \\
LIF & 450 & 420.689 & 1.50113 & $\mathrm{X}$ \\
Contrast & Sig. & Difference & + - Limits & \\
LIF - NEU & $*$ & 389.28 & 4.16085 & \\
\hline
\end{tabular}

*This pair shows significant differences at the $95 \%$ confidence level.

\section{Neuroid-based networks can replicate the firing patterns produced by short-scale networks composed of more detailed units}

Figures 6 and 7 show, respectively, the firing patterns produced by Neuroid-based replicas of both a three-neuron oscillator and the "cricket song generator". 
Table 3. Least-Squares Means for computation time with $95 \%$ confidence interval. Means, standard errors, lower and upper limits are expressed in milliseconds. An asterisk has been placed next to the factor showing a statistically significant difference between means at the $95 \%$ confidence level (LIF: leaky-integrate-and-fire; NEU: Neuroid).

\begin{tabular}{cccccc}
\hline Level & Count & Mean & Std. error & Lower limit & Upper limit \\
\hline $\begin{array}{c}\text { Grand mean } \\
\text { Model* }\end{array}$ & 900 & 226.049 & & & \\
LIF & 450 & 420.689 & 1.50113 & 417.747 & 423.632 \\
NEU & 450 & 31.4092 & 1.50113 & 28.467 & 34.3513 \\
Time step (ms)* & & & & \\
0.0001 & 180 & 974.001 & 2.37349 & 969.349 & 978.653 \\
0.001 & 180 & 133.477 & 2.37349 & 128.825 & 138.129 \\
0.01 & 180 & 19.5924 & 2.37349 & 14.9405 & 24.2444 \\
0.1 & 180 & 2.59033 & 2.37349 & -2.06164 & 7.2423 \\
1 & 180 & 0.58583 & 2.37349 & -4.06614 & 5.2378 \\
Stimulation period (ms) & & & & 223 \\
200 & 300 & 236.824 & 1.8385 & 233.221 & 240.427 \\
500 & 300 & 220.859 & 1.8385 & 217.256 & 224.463 \\
800 & 300 & 220.465 & 1.8385 & 216.861 & 224.068 \\
Stimulation amplitude (nA) & & & & \\
12 & 300 & 203.933 & 1.8385 & 200.33 & 207.536 \\
19 & 300 & 205.758 & 1.8385 & 202.154 & 209.361 \\
\hline
\end{tabular}

*This factor shows significant differences at the $95 \%$ confidence level.

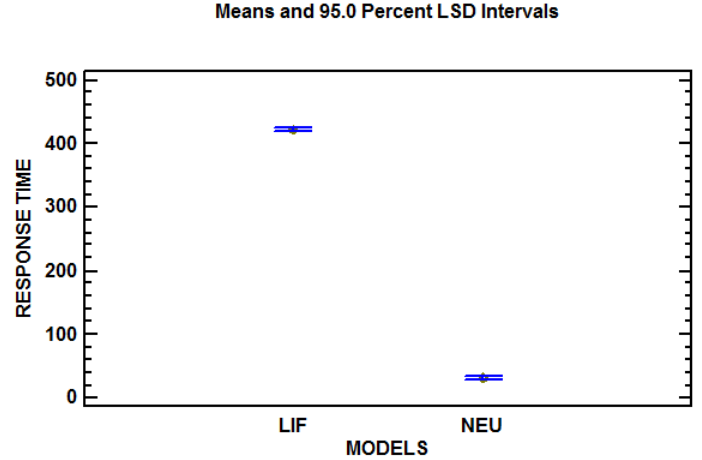

Figure 4. Least-Squares means of the computation times achieved by the LIF model (FE method) and the Neuroid. Both models were run for three different simulation amplitudes $(I=5,12$ and $19 \mathrm{nA})$ and for three different stimulation periods ( $T=200,500$ and $800 \mathrm{~ms}$ ), with time steps ranging from $10^{-4}$ to $1 \mathrm{~ms}$.

In general, results are in good agreement with those obtained by earlier studies, in which more detailed neuron-models were used (Friesen and Stent, 1977; Kling and Székely, 1968; Koch and Brunner, 1988). Different oscillation frequencies can be achieved by the Neuroid-based replica of the three neuron oscillator by varying the stimulation amplitude (see Figure $6 \mathrm{~b}$ ), and the order in which neurons start firing is also preserved $(\mathrm{N} 3 \rightarrow \mathrm{N} 2 \rightarrow \mathrm{N} 1 \rightarrow \mathrm{N} 3)$. Although the Neuroid-based replica of the "cricket song generator" required a transition period of more than $400 \mathrm{~ms}$ in order to produce a firing pattern consistent with previous results (see Figure 7b), Neuroids 2, 3 and 4 start firing shortly after the second spike of the previous unit occurs, and the burst of spikes

\section{Pareto Chart of the Standardized Effects (response is COMPUTATION TIME, $\alpha=0.05$ )}

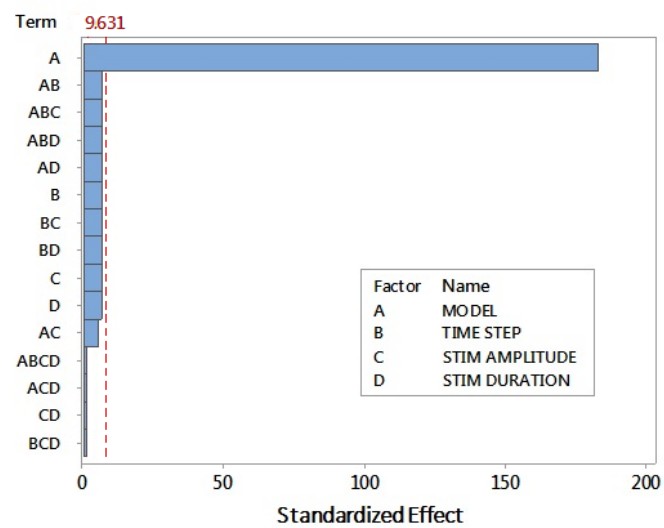

Figure 5. Absolute values of the standardized effects of each factor and all their possible combinations on the computation time. Only the model (A) has a significative and markedly effect on the computation time required to calculate several hundreds of milliseconds of neural response.

generated by $\mathrm{N} 5$ produces a long-term pause in N1, in consistency with previous results (see Figure 10 in Koch and Brunner, 1988).

\section{Discussion}

Figure 2a shows that, unlike the benchmark solution, the Neuroid was able to fire a single spike when the stimulus amplitude reached the threshold intensity $(I=3.7 \mathrm{nA})$, which is consistent with the differences 


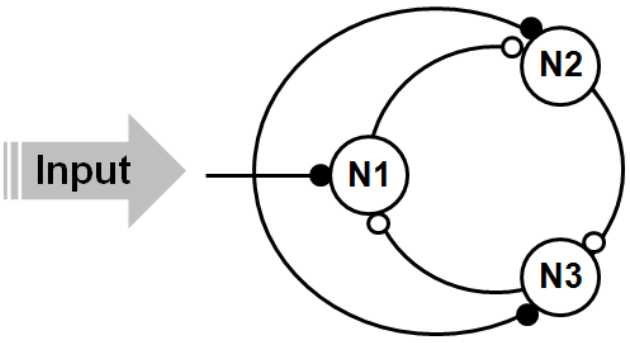

(a)
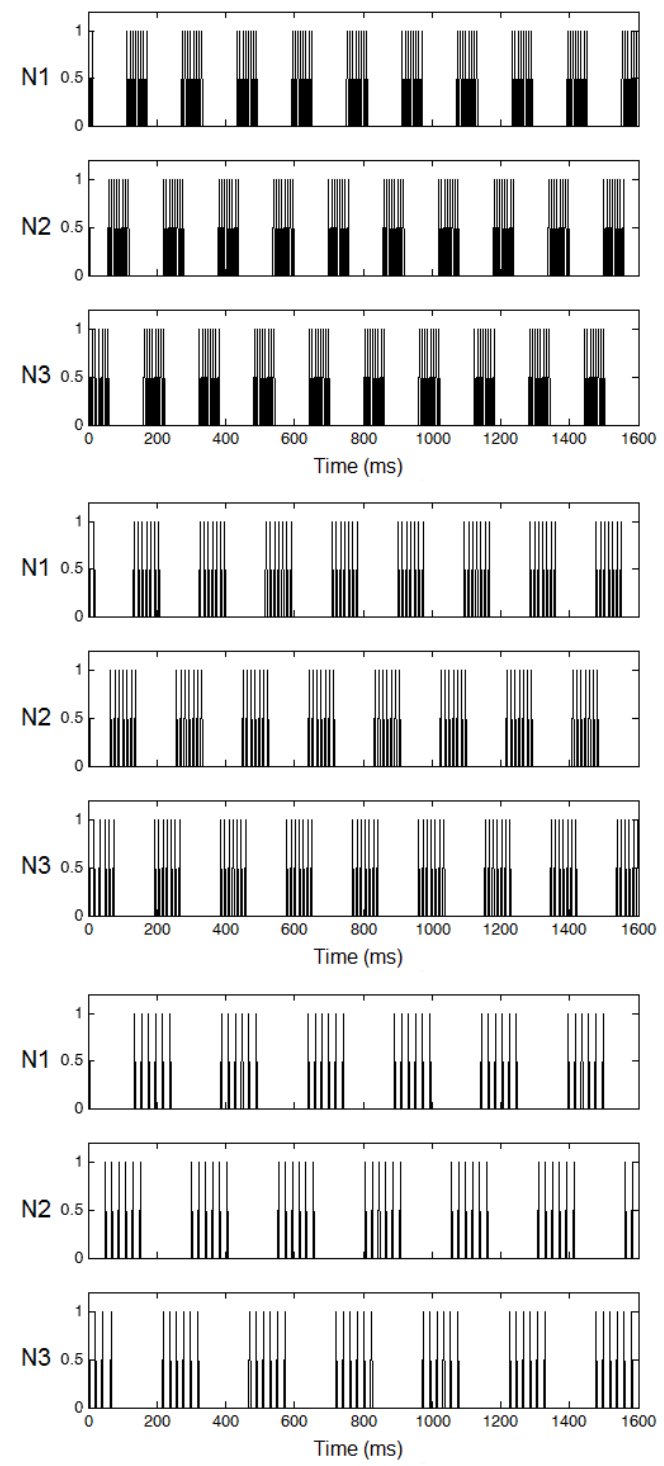

(b)

Figure 6. A three-Neuroid oscillator. (a) The oscillator consists of three neurons simultaneously receiving a constant excitatory input $(\bullet)$, while they are cyclically connected through inhibitory synapses (o). (b) Firing patterns obtained by the authors for three different simulation intensities (Input $=5,3$ and $2.7 \mu \mathrm{A}$ ) after implementing a Neuroid-based replica of the three-neuron oscillator.

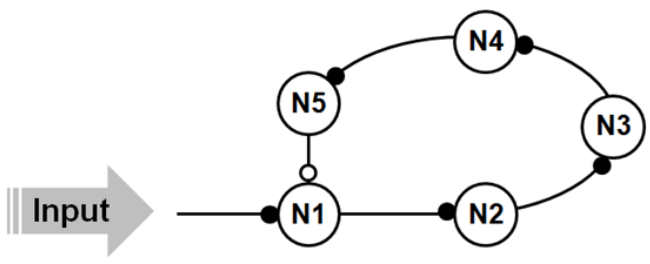

(a)
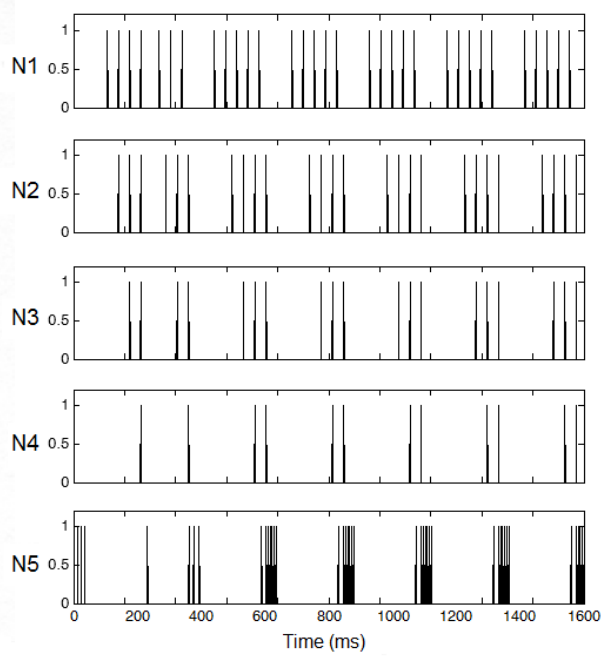

(b)

Figure 7. Using the Neuroid to build the "cricket song generator". (a) Neuron 1 receives a constant excitatory input, which is transmitted to neurons 2, 3, 4 and 5 through excitatory synapses $(\bullet)$, while neuron 5 provides neuron 1 with strong inhibition (०). (b) Firing patterns obtained by the authors after implementing a Neuroid-based replica of the "cricket song generator".

between the conditions required for the propagation of single spikes and those required for spike train generation (when sustained depolarizing pulses are applied at progressive increasing intensities, a "threshold intensity" is reached at which one single spike is fired... only when the stimulus intensity becomes higher, a spike train is produced) (Matzner and Devor, 1992). However, the response shown in Figure 2a cannot be assumed to be a phasic response, which include not only those exhibiting one single spike at the onset of the stimulus, but also those showing brief spike trains starting during the beginning of the stimulation period (Madrid et al., 2003; Mitra and Miller, 2007; Ruscheweyh and Sandkühler, 2002). In fact, the absence of a delay in spike firing at different stimulus intensities shows a fundamental limitation of our neuron-model and the assumption on which the model relies is, indeed, an oversimplification of the neural behavior because only some neurons fire at a regular frequency when stimulation amplitude is held constant and they do only under certain conditions. 
The Neuroid does not account for the dynamics of the voltage-dependent membrane conductances that are responsible for the generation of action potentials, which is why spike trains show no clear threshold dependence or temporal behavior consistent with the latencies of ionic channels (see Figure 2b). Small differences in the membrane voltage are a great concern for several neural phenomena, such as spike-timing-dependent plasticity (STDP) (Dan and Mu-ming, 2004; Shulz and Feldman, 2013), so the use and value of our neuron-model might be limited to a narrow band of applications.

While the Neuroid is now able to produce more accurate results when a time step of $10^{-2} \mathrm{~ms}$ or lower is used, its accuracy is considerably lower than the achieved with the LIF model solved by the FE method (see Table 1). The latter is consistent with the result obtained during a previous study (Skocik and Long, 2014), in which it was shown that the LIF model provides fairly accurate results when the FE method with a time step of $0.1 \mathrm{~ms}$ or lower is used. On the other hand, that comparison was carried out not between models but between numerical methods because, by definition, the firing frequency produced by one model is different from the firing frequency produced by the others. More reliable results could be obtained if the firing rate of the model is compared to the firing rate of a biological neuron. Still, this comparison would be a bit more complicated since the neural response varies between different populations. A previous study (Prada et al., 2015) found that the Neuroid might be used to predict the firing frequency of nociceptive primary afferent A- and C-fibers, but it could be insufficient to model spinal dorsal horn neurons. All this suggests that, in the absence of a quantitative index for the evaluation of the accuracy achieved by several neuron-models, the results obtained by previous (Izhikevich, 2004; Long and Fang, 2010; Skocik and Long, 2014; Wang et al., 2014) and the current study may not be absolutely conclusive.

From Figures 4 and 5, and Tables 2 and 3, it is clear that the computation time required by the Neuroid is significantly lower (Multifactorial ANOVA, $\mathrm{p}<0.05$ ) than the required by the LIF model when it is solved by using the FE method, which is one of the simplest methods for approximating solutions to differential equations (Izhikevich, 2004; Skocik and Long, 2014). In general, the Neuroid performs its calculations in an amount of time significantly lower than the required by the LIF model, under the same conditions. Surprisingly, such differences do not increase significantly either when the stimulation amplitude increases or the stimulation period decreases (see Table 3). Even so, it is worth noting that the FLOPs per iteration required by the Neuroid decreases by one whenever a spike is fired, since the variable count 1 is reset instead of increasing by one when its value reaches or exceeds the quantity $\frac{\beta}{(s(t)+\vartheta) \Delta t}$ (see Figure 1). Thus, as the stimulation amplitude increases and, therefore, the firing frequency, the total of FLOPs required for a given time of run decreases. Additionally, no operation is executed in the absence of stimulus because the Neuroid first compares the triggering event to the activation threshold and only if the former reaches or exceeds the latter, the model performs its calculations. On the contrary, classic neuron-models first update the membrane potential in terms of their parameters and the stimulus amplitude (even in the absence of stimulus), and then compare the resulting potential to the activation threshold, after which a spike can or cannot be fired (Gerstner et al., 2014). The ideas above are summarized in Figure 8.

This "compare first, calculate later" feature makes the Neuroid an attractive option for those who want to model the behavior of large-scale neural networks through which the information is transmitted in the form of short-duration pulses (e.g., Spiking Neural Networks), especially when computational resources are limited, since its computational cost is significantly lower than the one required by the legendary LIF model. For instance, about one third to $40 \%$ of the cortical neuron population is composed by neurons capable of firing extremely narrow spikes at a very high frequency $(300-400 \mathrm{~Hz})$ with a very low adaptation rate (Wang et al., 2016), precisely the kind of behavior that the Neuroid is able to reproduce. However, it must be taken into account that the computational cost of a neuron-model tends to increase with its accuracy, which in turn depends on, among other factors, the size of the time step. Nowadays, it is possible to implement some alternatives, like the one proposed by Wang et al. (2014), whose accuracy is not significantly affected by the time step size. Yet, and as we previously pointed out, results reported by that and other studies should be analyzed in the light of a common quantitative index of a neuron-model's accuracy, which may lead to a more objective comparison.

Figures 6 and 7 show that it is feasible to use Neuroid-based networks to replicate biologically relevant firing patterns produced by low-scale networks composed of much more detailed units, as those used in earlier studies (Friesen and Stent, 1977; Kling and Székely, 1968; Koch and Brunner, 1988). Moreover, it was possible to generate periodic bursts of spikes by using a heuristic that was originally conceived to respond tonically. No matter which values are chosen for the parameters of the Neuroid, it is not able to produce by itself bursts of spikes, unlike other spiking neuron-models (e.g., the Izhikevich's model). This suggests that, even though spike bursts are known to be caused by intrinsic mechanisms of cells (Izhikevich, 2007; Krahe and Gabbiani, 2004), they can also be attributed to the synaptic properties of 


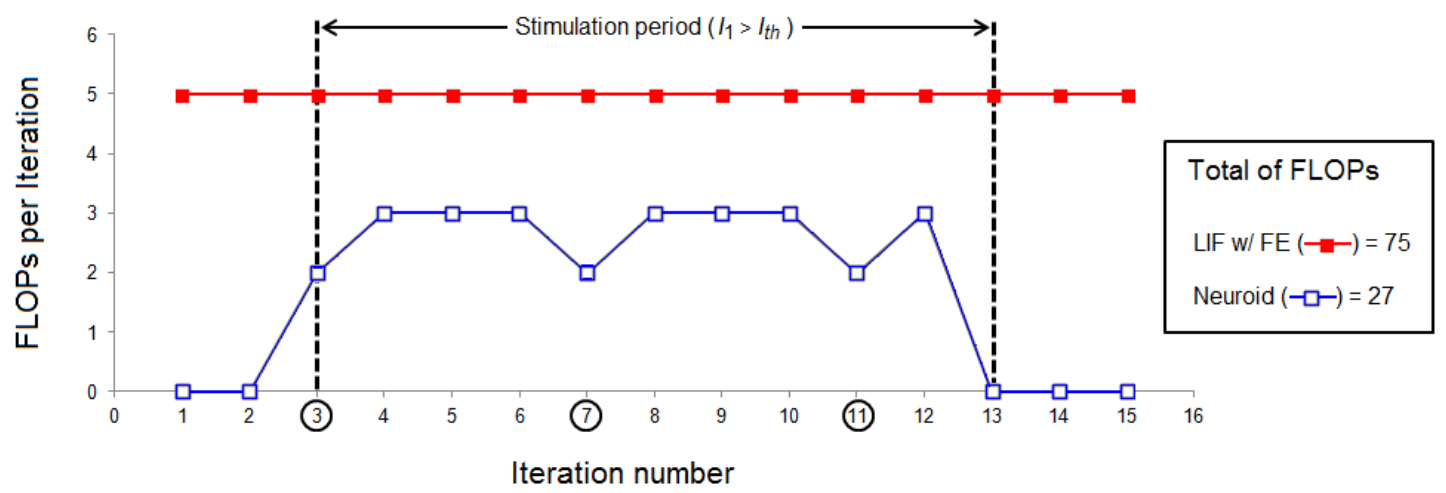

(a)

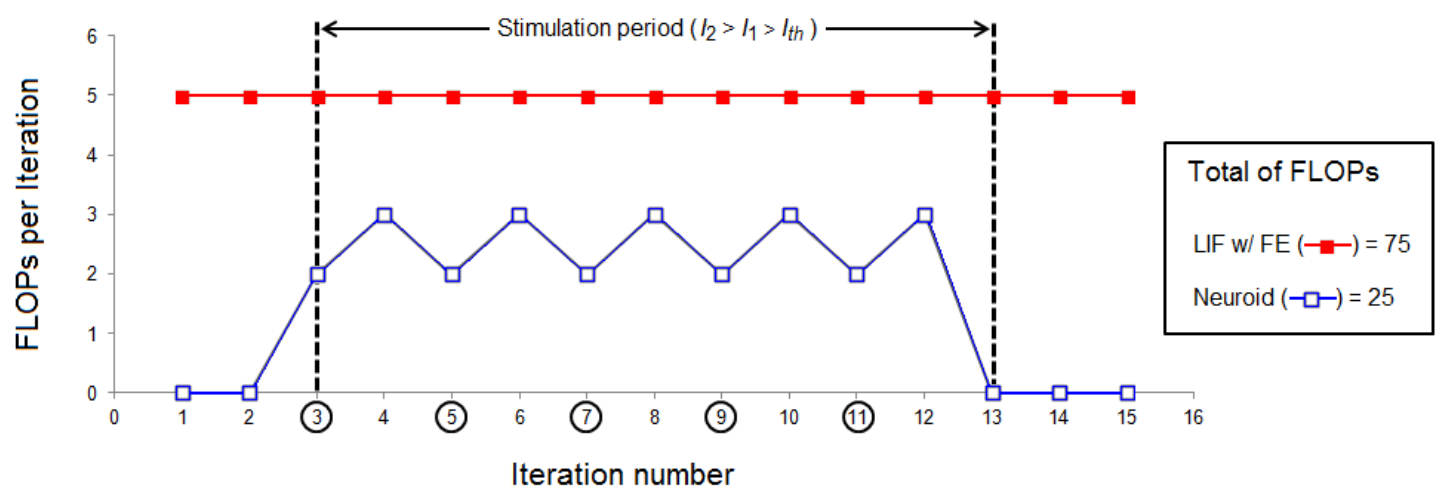

(b)

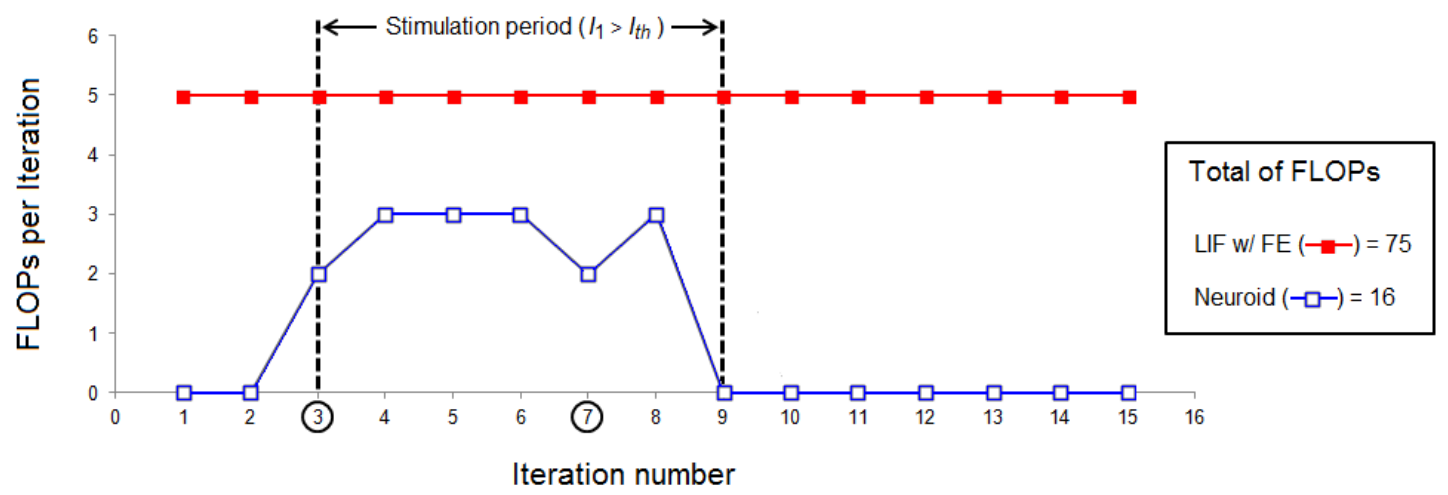

(c)

Figure 8. Effects of the stimulus amplitude and duration on the estimated computational cost of the Neuroid and the LIF model solved by the FE method. The band between dashed lines denotes the stimulation period and an encircled number indicates that a spike was fired. It was assumed that both models fire synchronously. (a) The total of FLOPs required by the Neuroid to perform its calculations is, in general, less than that for the LIF model when it is solved by using the FE method because no operations are executed by the Neuroid in the absence of stimulus; (b) As the stimulus amplitude increases, the FLOPs per iteration required by the Neuroid decreases by one per each "elicited" spike and the FLOPs required for the given total of iterations decreases slightly; (c) As the stimulus duration decreases, the FLOPs required by the Neuroid for the given total of iterations decreases considerably because the FLOPs per iteration drops to zero in the absence of stimulus. On the contrary, the LIF model (like other classic neuron-models) is constantly performing calculations regardless the amplitude and duration of the stimulus, so the FLOPs per iteration required by the model and the FLOPs for the given total of iterations remains unaffected, even in the absence of stimulus. 
neuron connectivity and/or the topology of the network. Therefore, it would be possible to use Neuroids in analyzing to what extent emergent properties derived from mutual connectivity between neurons contribute to the generation of bursting oscillatory patterns. Neuroid-based replicas of some biological neural networks, whose architecture is well known, might be built and studied before using much more detailed simulation tools or packages. This, in turn, demands an accurate identification of the cells composing those networks as well as a wiring diagram describing how they are connected, which may lead to new research opportunities. Lastly, a result like this could be viewed as a small extension of the Hopfield's work (Hopfield, 1984; 1982), in which it was found that the computational properties of networks composed of binary unrealistic units, like the McCulloch and Pitts model (McCulloch and Pitts, 1943), are often preserved in networks composed of units having continuous input-output relationships and integrative time delays.

Like any heuristic, the Neuroid does not guarantee an optimal solution for a wide range of problems. Our neuron-model is, indeed, far from being a biologically meaningful model, since the conditions and assumptions upon which the model was formulated are very specific. It is widely known that the firing rate of real neurons depends not only on the stimulation intensity, but also on several other factors like subthreshold dynamics, a feature which our neuron-model fails to exhibit. On the other hand, and according with results presented before, the Neuroid requires a smaller amount of computational resources than those required by the LIF model to simulate several hundreds of milliseconds of neuronal response under the same conditions. This suggests that the Neuroid may be an interesting option for the implementation of networks through which the information is transmitted in the form of short-duration pulses (e.g., Spiking Neural Networks), especially when computational resources are limited. Furthermore, the low computational cost required by the Neuroid, as well as its flexibility to be adapted to any type of programming language $(\mathrm{C}++$, MATLAB, HPVEE, LabVIEW) make it suitable for the development of free and open computational tools based on Neuroids, as the one developed in the University of Guayaquil (Silva Muñoz, 2015). Future work may include considering the noise influence by adding a probabilistic component to the Neuroid without altering its low computational requirement.

The fact that our neuron-model is able to reproduce only a few neuro-computational properties could make it a useful tool for determining if the firing patterns exhibited by neurons composing certain networks are due to the intrinsic properties of these cells, or to the topology of the network. In other words, if a Neuroid-based replica is able to reproduce the firing patterns observed in a biological network, then these patterns are likely to be produced by the interactions between different cells or nodes. This indeed underlines the importance of neural connection for the generation of specific firing patterns, and also demands an accurate identification of the cells composing those networks as well as a wiring diagram describing how they are connected, which may lead to new research opportunities.

As a concluding remark, beyond a simple comparison between different average firing rates or coincident spikes, researchers and modelers still lack a standardized set of tests to quantify and compare the accuracy of distinct neuron-models, so further work is needed to provide these communities with a common framework that can be used as a reference for this purpose.

\section{Acknowledgements}

The authors thank Prof. Minaya Villasana for her valuable ideas and useful observations during the realization of this article.

\section{References}

Alberts B, Johnson A, Lewis J, Raff M, Roberts K, Walter P. Molecular biology of the cell. 4th ed. New York: Garland Science; 2002.

Andrews T. Computation time comparison between Matlab snd C++ using Launch Windows. Aerosp Eng. 2012; 78:1-6.

Bayly EJ. Spectral analysis of pulse frequency modulation in the nervous systems. IEEE Trans Biomed Eng. 1968; 15(4):257-65. PMid:5699902. http://dx.doi.org/10.1109/ TBME.1968.4502576.

Brette R, Gerstner W. Adaptive exponential integrate-andfire model as an effective description of neuronal activity. $\mathrm{J}$ Neurophysiol. 2005; 94(5):3637-42. PMid:16014787. https:// doi.org/ 10.1152/jn.00686.2005.

Dan Y, Mu-ming P. Spike timing-dependent plasticity of neural circuits. Neuron. 2004; 4(1):23-30. https://doi.org/10.1016/j. neuron.2004.09.007.

Delcomyn F. Neural basis of rhythmic behavior in animals. Science. 1980; 210(4469):492-8. PMid:7423199. http://dx.doi. org/10.1126/science.7423199.

Ermentrout GB, Terman DH. Mathematical foundations of neuroscience. Vol. 35. New York: Springer Science \& Business Media; 2010

Friesen WO, Stent GS. Generation of a locomotory rythm by a Neural Network with Recurrent Cyclic Inhibition. Biol Cybern. 1977; 28(1):27-40. PMid:597508. http://dx.doi. org/10.1007/BF00360911.

Gerstner W, Kistler WM, Naud R, Paninsky L. Neuronal dynamics: from single neurons to networks and models of cognition. Cambridge: University Press; 2014.

Hodgkin AL, Huxley AF. A quantitative description of membrane current and its application to conduction and excitation in 
nerve. J Physiol. 1952; 117(4):500-44. PMid:12991237. http:// dx.doi.org/10.1113/jphysiol.1952.sp004764.

Hopfield JJ. Neural networks and physical systems with emergent collective computational abilities. Proc Natl Acad Sci USA. 1982; 79(8):2554-8. PMid:6953413. http://dx.doi. org/10.1073/pnas.79.8.2554.

Hopfield JJ. Neurons with graded response have collective computational properties like those of two-state neurons. Proc Natl Acad Sci USA. 1984; 81(10):3088-92. PMid:6587342. http://dx.doi.org/10.1073/pnas.81.10.3088.

Horch KW, Dhillon GS. Neuroprosthetics: theory and practice. New York: World Scientific; 2004.

Izhikevich EM. Simple model of spiking neuron. IEEE Trans Neural Netw. 2003; 14(6):1569-72. PMid:18244602. https:// doi.org/10.1109/TNN.2003.820440.

Izhikevich EM. Which model to use for cortical spiking neurons? IEEE Trans Neural Netw. 2004; 15(5):1063-70. PMid:15484883. https://doi.org/10.1109/TNN.2004.832719.

Izhikevich EM. Dynamical systems in neuroscience: the geometry of excitability and bursting. Cambridge: MIT Press; 2007.

Jolivet R, Lewis TJ, Gerstner W. Generalized integrate-andfire models of neuronal activity approximate spike trains of a detailed model to a high degree of accuracy. J Neurophysiol. 2004; 92(2):959-76. PMid:15277599. https://doi.org/10.1152/ jn.00190.2004.

Kling U, Székely G. Simulation of rhythmic nervous activities. I. Function of networks with cyclic inhibitions. Kybernetik. 1968; 5(3):89-103. PMid:5728516. http://dx.doi.org/10.1007/ BF00288899.

Kobayashi R, Tsubo Y, Shinomoto S. Made-to-order spiking neuron model equipped with a multi-timescale adaptive threshold. Front Comput Neurosci. 2009; 3:9. PMid: 19668702. https:// doi.org/10.3389/neuro.10.009.2009.

Koch UT, Brunner M. A modular analog neuron-model for research and teaching. Biol Cybern. 1988; 59(4-5):303-12. PMid:3196775. http://dx.doi.org/10.1007/BF00332920.

Krahe R, Gabbiani F. Burst firing in sensory systems. Nat Rev Neurosci. 2004; 5(1):13-23. PMid:14661065. http://dx.doi. org/10.1038/nrn1296.

Long L, Fang G. A review of biologically plausible neuron models for spiking neural networks. In: Proceedings of the AIAA InfoTech@Aerosp Conference; 2010Apr 20-22; Atlanta, GA. Atlanta:AIAA; 2010. http://dx.doi.org/10.2514/6.2010-3540.

Madrid R, Sanhueza M, Alvarez O, Bacigalupo J. Tonic and phasic receptor neurons in the vertebrate olfactory epithelium. Biophys J. 2003; 84(6):4167-81. PMid:12770919. https://doi. org/10.1016/S0006-3495(03)75141-8.

Matzner O, Devor M. Na+ conductance and the threshold for repetitive neuronal firing. Brain Res. 1992; 597(1):92-8. PMid:1335824. http://dx.doi.org/10.1016/0006-8993(92)91509-D.

McCulloch WS, Pitts WH. A logical calculus of the ideas immanent in nervous activity. Bull Math Biol. 1943; 5(4):11533. https://doi.org/ 10.1007/BF02478259.
Mitra P, Miller RF. Normal and rebound impulse firing in retinal ganglion cells. Vis Neurosci. 2007; 24(1):79-90. PMid:17430611. https://doi.org/10.1017/S0952523807070101.

Montgomery DC. Design and analysis of experiments. Hoboken: John Wiley \& Sons; 2017.

Prada EJA, Bustillos RJS, Huerta MK, Martínez ADA. Lamina specific loss of inhibition may lead to distinct neuropathic manifestations: a computational modeling approach. Res Biomed Eng. 2015; 31(2):133-47. http://dx.doi.org/10.1590/24464740.0734 .

Prada EJA, Bustillos RJS, Castillo C, Huerta M. New trends in computational modeling: a neuroid-based retina model. In: Proceedings of the 35th Annual International Conference IEEE Engineering in Medicine and Biology Society; 2013 Jul 3-7; Osaka, Japan. Osaka: IEEE; 2013. p. 4561-4. PMid: 24110749. https://doi.org/10.1109/EMBC.2013.6610562.

Prada EJA, Bustillos RJS, Castillo C, Huerta M. The neuroid: a novel and simplified neuron-model. In: Proceedings of the 34th Annual International Conference IEEE Engineering in Medicine and Biology Society; 2012 Aug 28-Sep 1; San Diego, CA. San Diego: IEEE; 2012. p. 1234-7. PMid:23366121. https://doi.org/10.1109/EMBC.2012.6346160.

Prada EJA, Bustillos RJS. The implementation of the Neuroid in the gate control system leads to new ideas about pain processing. Rev Bras Eng Bioméd. 2013; 29(3):254-61. http:// dx.doi.org/10.4322/rbeb.2013.025.

Rieke F, Warland D, van Steveninck RR, Bialek W. Spikes: exploring the neural code. Cambridge: MIT Press; 1997.

Ruscheweyh R, Sandkühler J. Lamina-specific membrane and discharge properties of rat spinal dorsal horn neurones in vitro. J Physiol. 2002; 541(1):231-44. PMid:12015432. http:// dx.doi.org/10.1113/jphysiol.2002.017756.

Silva Muñoz AM. Desarrollo de un software para la construcción de redes neuroidales [thesis]. Guayaquil-Ecuador: Universidad de Guayaquil; 2015.

Scimemi A, Beato M. Determining the neurotransmitter concentration profile at active synapses. Mol Neurobiol. 2009. 40(3):289-306. https://doi.org/ 10.1007/s12035-009-8087-7.

Shulz DE, Feldman DE. Spike timing-dependent plasticity. In: Rubenstein JLR, Rakic P. Comprehensive developmental neuroscience: neural circuit development and function in the healthy and diseased brain. San Diego, CA: Elsevier; 2013. p. $155-81$.

Skocik MJ, Long LN. On the capabilities and computational costs of neuron models. IEEE Trans Neural Netw Learn Syst. 2014; 25(8):1474-83. PMid: 25050945. https://doi.org/10.1109/ TNNLS.2013.2294016.

Wang Z, Guo L, Adjouadi M. A generalized Leaky Integrateand-Fire neuron model with fast implementation method. Int J Neural Syst. 2014; 24(5):1440004. PMid:24875788. http:// dx.doi.org/10.1142/S0129065714400048.

Wang B, Ke W, Guang J, Chen G, Yin L, Deng S, et al. Firing frequency maxima of fast-spiking neurons in human, monkey, and mouse neocortex. Front Cell Neurosci. 2016; 10:239. http:// dx.doi.org/10.3389\%2Ffncel.2016.00239. 